\title{
La répartition des responsabilités
}

\author{
J. GONNET ${ }^{\mathrm{a}, 1}$, G. ABÉLA ${ }^{2}$, S. BÉRARD ${ }^{3}$, P. BOURDON $^{4}$, O. COUASNON $^{5}$, \\ M. FELTZINGER ${ }^{6}$, E. MARTIN ${ }^{7}$, G. PERRAUDIN $^{8}$, \\ Y. TAFFARD ${ }^{9}$, R. VENNIN ${ }^{10}$
}

RÉSUMÉ Ce document est un guide pour les entreprises utilisant ou réalisant pour des clients des Contrôles ou Examens Non Destructifs. Il reprend l'ensemble des étapes de ce type de prestations (sur la base d'un travail déjà réalisé au niveau de la région PACA) et identifie la répartition des responsabilités entre : entreprise utilisatrice (EU)/ donneur d'ordre, ensemblier ou maître d'œuvre (MOE) et entreprise intervenante (EI) en radiographie. Il rappelle certaines obligations et propose de «bonnes pratiques " recueillies auprès d'industriels du domaine. Il convient d'abord de préciser les points forts qui définissent cette répartition de responsabilités. Celle-ci s'appuie en premier lieu sur une relation contractuelle entre les 2 ou 3 parties prenantes. L'entreprise utilisatrice confie ainsi à l'ensemblier/maître d'œuvre et luimême à l'entreprise intervenante les responsabilités de faire réaliser et/ou de réaliser les prestations dans le respect des exigences réglementaires et dans les conditions de sécurité optimum. Chaque entité assure donc ses responsabilités, ceci étant la base fondamentale et incontournable de leur implication pertinente dans la tenue des objectifs opérationnels et de sécurité. Cette relation contractuelle se construit en particulier à certaines étapes clés : l'identification précise des objectifs et conditions de réalisation (déclinaison des exigences) lors de l'élaboration du cahier des charges ; identification des éléments devant être précisés pour maîtriser l'intervention lors de la revue d'offre ; l'acceptation ou l'identification des réserves à lever lors de la revue de commande ; l'identification nominative des personnes responsables du contrat et des travaux dans chaque entité ; et enfin l'élaboration du plan de prévention. Dans certains cas, pour la recherche des informations nécessaires au cahier des charges, l'élaboration du plan de prévention, la détermination des conditions radiologiques, la définition de la zone d'opération, etc., l'entité concernée peut, faute de compétences existantes ou disponibles, faire appel au conseil et support de sociétés compétentes. Cela ne la dégage pas de ses responsabilités mais peut lui permettre de répondre efficacement aux exigences à satisfaire. NOTA : en fonction des conseils proposés, si l'entité ne souhaite pas suivre les recommandations, elle doit le signifier clairement et la société d'assistance lui rappeler ses obligations. De plus, l'entité qui sous-traite une partie de ses activités s'assure de la bonne réalisation par la surveillance qu'elle juge utile. Enfin, le retour d'expérience est une démarche qui, faite par l'entreprise intervenante ou capitalisée par l'entreprise utilisatrice, est de nature à améliorer les

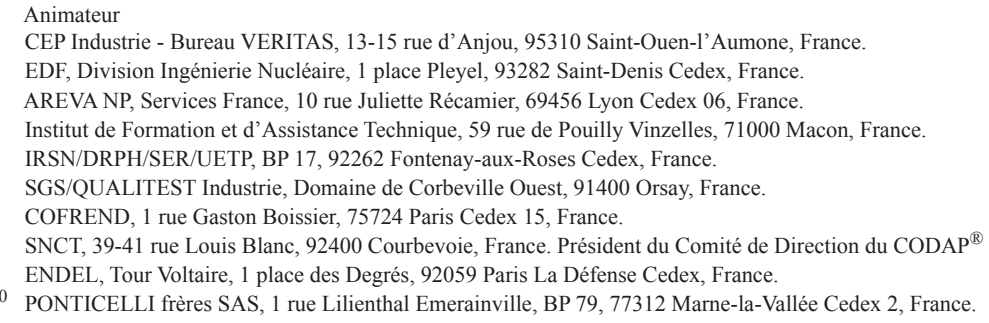


conditions de réalisation dans le temps dès qu'il est réutilisé comme données d'entrée pour de nouvelles interventions.

ABSTRACT The distribution of the responsabilities.

This document is a guide for the companies using or performing for customers Non Destructive Testings. It takes again the whole of the stages of this type of services (on the basis of work already completed on the level of "Région PACA in France") and identifies the distribution of the responsibilities between: Customer Cie (EU), Project superintendent (MOE), and intervening company (I.E) in radiography. It points out certain obligations and proposes "good practices" received from industrialists of the field. It is initially advisable to clarify the strong points which define this distribution of responsibilities. This one is based initially on a contractual relation between the 2 or 3 fascinating parts. The Company User thus entrusts to the ensemblier/project superintendent and itself with the intervening company the responsibilities to make realize and/or to carry out the services in the respect of the lawful requirements and the conditions of optimum safety. Each entity thus ensures its responsibilities, this being the base fundamental and impossible to circumvent of their relevant implication in the behaviour of the operational objectives and of safety.

Keywords: Radiography / responsability

\section{Légende}

R1 : Responsable

$\mathrm{R} 2$ : Responsable

$\mathrm{C}:$ Contribue, Participe

$\mathrm{V}$ : Vérifie

I : Informe (via le coordinateur de chantier le cas échéant)

$\mathrm{EU}=$ entreprise utilisatrice (exploitant du site)

MOE = maître d'œuvre

$\mathrm{EI}=$ entreprise intervenante

Nota : dans le cas d'une commande en direct EU/EI la responsabilité RI de l'EU est complète; dans les cas d'une commande à un maître d'ouvre/ensemblier, celui-ci assume également une part de responsabilité $R 2$. 


\begin{tabular}{llccc}
\hline \multicolumn{1}{|c}{ Étapes } & $\begin{array}{c}\text { Entreprise } \\
\text { Utilisatrice } \\
\text { (exploitant } \\
\text { du site) }\end{array}$ & $\begin{array}{c}\text { Maître } \\
\text { d'œure }\end{array}$ & $\begin{array}{c}\text { Entreprise } \\
\text { interve- } \\
\text { nante }\end{array}$ & Observations \\
\hline 1. Pré-requis à la préparation des travaux & EU & MOE & EI & \\
\hline 1.1 Élaboration du cahier des charges & EU & MOE & EI
\end{tabular}

Le donneur d'ordre procède à l'analyse des besoins en identifiant :

- Le cadre de l'opération à réaliser (maintenance, urgence...)

- La nature des contrôles (soudure, épaisseur, type de dégradations recherchées, recherche de corps étranger...)

- Le diamètre, épaisseur, matière, ...

- Les lieux des interventions (sur site industriel, atelier...) Identification précise de la ou des zones à inspecter et des équipements existant sur place

- Les dates (période couverte précisant date de début et de fin prévisible)

- Les horaires des tirs (plage horaire indicative). Le travail en journée (voire décalé en fin de journée) doit être privilégié par rapport au travail de nuit

- Les procédures spécifiques du lieu des tirs (ex : activité maximum de la source autorisée sur le site industriel) ou référence (procédure, $\mathrm{N}^{\circ} \mathrm{d}$ 'ordre avec date de révision, dernier indice de révision)

- L'environnement de travail : les risques spécifiques (bruit, éclairage, travail en moyenne et grande hauteur, travail confiné, froid, cartographie (Irradiation et contamination), co-activités prévisionnelles, ...) et les mesures de prévention adaptées en précisant qui en a la responsabilité. Certaines conditions de travail seront susceptibles de conduire au choix d'une méthode alternative de contrôle.

Les documents à produire (permis de tir, fiche d'intervention, fiche d'urgence, compte-rendu d'intervention, ...)

\subsection{Consultation}

EU

MOE

EI

\section{Le choix des entreprises intervenantes s'effectuera :}

Selon les critères propres à l'entreprise (Certification, habilitation, ...) en tenant compte des compétences en prévention manifestées.
R1 R2
La participation de l'EI à l'élaboration de certains documents peut être bénéfique, notamment le planning prévisionnel avec plusieurs scénarii éventuellement.

En cas de co-activités, une coordination spécifique peut être efficace.
Il est préférable de sélectionner si possible une seule entreprise de radiographie industrielle pour l'ensemble des tirs à réaliser 


\begin{tabular}{llcc}
\hline Étapes & $\begin{array}{c}\text { Entreprise } \\
\text { Utilisatrice Maître } \\
\text { (exploitant d'ouvre } \\
\text { du site) }\end{array}$ & $\begin{array}{c}\text { Entreprise } \\
\text { interve- } \\
\text { nante }\end{array}$ & Observations \\
\hline 1.3 Offre & EU & MOE & EI
\end{tabular}

Au Cahier des Charges, l'EI fait une offre

d'intervention en proposant les moyens nécessaires

(techniques et humains) à l'atteinte des objectifs en ayant décliné :

> Le procédé de contrôle,

- L'organisation mise en place,

$>$...,

(dans la suite du texte, on suppose que le procédé

de contrôle proposé est la radiographie industrielle)

\subsection{Commande}

La commande sera passée selon les modalités propres à l'entreprise.

Aucune intervention ne sera possible sans bon de commande.
E

R1

R2

C'est à l'EI de ne rien

faire si elle n'a pas de commande.

Cependant,

l'intervention

peut avoir été

complètement préparée

(ressources, matériels,

démarches, ...) par

$\mathbf{R}$

I'EI à ses frais et san

compensation si la

commande n'est pas

émise dans les temps.

L'EI pourrait exiger une intention de commande dans un délai de 2 à $5 \mathrm{j}$ avant l'intervention sans quoi elle en suspend la préparation.

Le plan de prévention, obligatoirement écrit dans le cas de l'utilisation des rayonnements ionisants, est établi par le chef de l'entreprise utilisatrice et formalise après une inspection commune préalable à l'exécution de l'opération, l'analyse des risques professionnels, les mesures de prévention nécessaires ainsi que le suivi de toute intervention réalisée par une ou plusieurs entreprises extérieures.

Les services de contrôle (Inspections du Travail et de la Radioprotection) doivent être informés préalablement des programmes de tirs. 


\section{Étapes}

Entreprise
Utilisatrice Maître
(exploitant d'œuvre
du site)

Entreprise

interve-

Observations

Pour les travaux de gammagraphie de plus de 30 jours consécutifs, l'entreprise de radiographie doit effectuer, de plus, une déclaration auprès du préfet du département dans lequel le chantier est prévu et de l'autorité ayant délivré l'autorisation (ASN ou inspection des installations classées).

La fiche d'intervention ou le permis de tirs constitue un élément du Plan de prévention. Il est déclenché par le donneur d'ordre. L'élaboration d'une fiche d'intervention est systématique et propre à chaque opération.

Elle doit notamment s'appuyer sur les informations recueillies lors d'une visite commune (donneur d'ordre et entreprises intervenantes) qui devra avoir lieu préalablement à la réalisation des travaux.

Cette fiche fait partie intégrante du plan de prévention qu'elle vient compléter ou préciser au niveau du détail de l'opération mais ne s'y substitue pas.

Elle est déclenchée par le donneur d'ordre au moment de la commande.

\subsection{Le donneur d'ordre}

Il renseigne, a minima, en coordination avec sa PCR (si INB ou ICPE) ou son chargé de sécurité (hors INB) :

- Le travail à effectuer (programme des tirs, types de tirs, atelier, site...)

- Le plan des zones et la localisation des points des tirs

- Les caractéristiques spécifiques du travail à réaliser et pouvant nécessiter des moyens techniques et de protections particuliers (échafaudage adapté, éclairage, ...)

- Les éléments de sécurité (cheminements et sorties de secours, téléphones, boites à pharmacie, extincteurs, moyens d'urgence, douche/rince œil, ...). Pour les sites industriels, ces éléments qui peuvent évoluer jusqu'au dernier moment, seront confirmés par le document de mise au travail

- La conduite à tenir en cas d'incident, le numéro de téléphone du DO à contacter (sur site industriel c'est le chef de quart, en atelier une personne est à désigner), les informations spécifiques essentielles de la sécurité (ambiance de travail « chaud, froid, ... », conduite à tenir en cas d'accident, risque généré par le radiologue industriel)

- Tout élément jugé utile pour une bonne réalisation des travaux sans alourdir exagérément les documents liés à l'opération.

R C V

S'assurer de la correspondance pour des bâtiments semblables. Dans le cas où le chargé de sécurité $\mathrm{C}$
et/ou et/ou exemple ne connait pas tous les aspects relatifs à la radioprotection, il se doit de solliciter une assistance de l'EI ou d'un autre prestataire. 


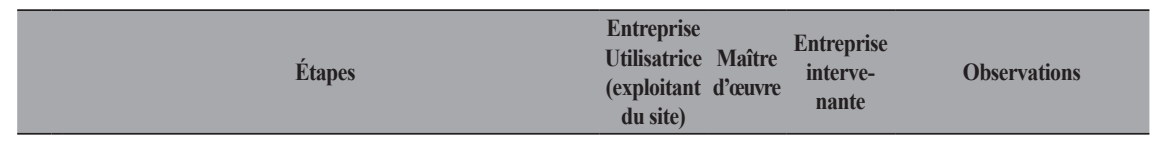

Renseigne, a minima, en coordination avec sa PCR ou

son chargé de sécurité (hors INB) :

- Le travail à effectuer (programme des tirs, types de tirs, atelier, site...)

- Le plan des lieux d'intervention et la localisation des points de tirs (à l'aide, par exemple, d'une signalétique de couleur ou d'étiquettes spécifiques).

- Les caractéristiques spécifiques du travail à réaliser et pouvant nécessiter des moyens techniques et de protections particuliers :

a) échafaudage adapté, présentant une accessibilité correcte, tenant compte de l'utilisation d'un matériel lourd et encombrant et de la nécessité de se déplacer rapidement sur des points de replis,

b) éclairage particulier (adapté au travail nocturne ou aux zones mal éclairées)

c) chaleur, bruit...

- Les éléments essentiels de sécurité existants (cheminements et sorties de secours, téléphones, boites à pharmacie, extincteurs, moyens d'urgence, douche/rince œil...).

- (Pour les sites industriels, ces éléments qui peuvent évoluer jusqu'au démarrage des travaux, seront confirmés par le document de mise au travail qui devra être annexé au plan de prévention)

- La conduite à tenir en cas d'incident, le numéro de téléphone du donneur d'ordres à contacter (sur site industriel, c'est le chef de quart ou représentant de l'industriel ; en atelier, une personne est à désigner par le chef d'entreprise).

- Les informations spécifiques essentielles de la sécurité (ambiance de travail « chaud, froid », conduite à tenir en cas d'accident, risques générés par le radiologue industriel, risques générés par le donneur d'ordres dans le cadre de son activité).

- Tout élément jugé utile pour une bonne réalisation des travaux (risque d'interférence avec d'autres équipes de radiologues ou d'autres intervenants) sans alourdir exagérément la fiche d'intervention qui doit rester un document pratique.

\subsection{L'entreprise de radiographie industrielle}

Elle renseigne:

La partie dosimétrie prévisionnelle et le balisage : Elle indique sur les plans d'installations fournis les limites de la «zone d'opération » (si possible $48 \mathrm{~h}$ avant les tirs*) Elle identifie le nom de la PCR pouvant intervenir ainsi que son numéro de téléphone.
S'assurer de la correspondance pour des bâtiments semblables.

Dans le cas où le

C chargé de sécurité

et/ou (petites structures par

$\mathrm{V}$ exemple ne connait pas tous les aspects relatifs à la radioprotection, $i l$ se doit de solliciter une assistance de l'EI ou d'un autre prestataire. 


\begin{tabular}{lll} 
Étapes & $\begin{array}{l}\text { Entreprise } \\
\text { Utilisatrice Maître } \\
\text { (exploitant d'œuvre } \\
\text { du site) }\end{array}$ & $\begin{array}{c}\text { Entreprise } \\
\text { interve- } \\
\text { nante }\end{array}$ \\
\hline
\end{tabular}

Renseigne :

- La partie dosimétrie prévisionnelle, la zone de balisage ou d'opération (48 $\mathrm{h}$ avant les tirs)

- Le nom et les coordonnées téléphoniques de la PCR pouvant intervenir,

- La liste du matériel utilisé

- Les doses reçues en fin d'opération, sous réserve que le chef d'entreprise s'assure de la confidentialité des données nominatives

- Le retour d'expérience (REX)

La fiche d'intervention doit être visée par le donneur

d'ordre et le radiologue industriel.

Le permis de tir doit être visé par le donneur d'ordre et le radiologue industriel.

R1 R2
Elle doit avoir en sa possession toutes les informations nécessaires à la préparation de ses travaux. Si elle estime ne pas disposer de toutes les informations nécessaires tant sur le plan technique que sécurité, elle doit le signaler et demander, les compléments d'informations nécessaires.

Une visite préalable de chantier avec le DO est recommandée pour préciser les conditions d'interventions.

\author{
* Pour les petites \\ structures et/ou \\ missions ponctuelles, le \\ délai est souvent plus \\ court
}

$\mathbf{R}$

Par sa signature, l'entreprise intervenante s'engage à appliquer la réglementation et les règles de l'art et acte qu'elle en a les moyens (intervenants CAMARI pouvant adapter le balisage si besoin, responsable d'intervention désigné de l'équipe)

Des situations de travail différentes peuvent être rencontrées en radiographie industrielle en fonction du type de chantier (sur site industriel, en atelier, à l'ensemble de ces situations sont présentées ci-dessous. Les mesures particulières sont détaillées dans les paragraphes correspondants. 


\begin{tabular}{|c|c|c|c|c|}
\hline Étapes & $\begin{array}{c}\text { Entreprise } \\
\text { Utilisatrice } \\
\text { (exploitant } \\
\text { du site) }\end{array}$ & $\begin{array}{l}\text { Maître } \\
\text { t'œuvre }\end{array}$ & $\begin{array}{c}\text { Entreprise } \\
\text { interve- } \\
\text { nante }\end{array}$ & Observations \\
\hline
\end{tabular}

L'employeur

- choisit le mode opératoire, le nombre d'équipes, le matériel adapté (check list du matériel d'intervention et du matériel de protection) et s'assure, par les visites périodiques et le suivi du matériel, de son bon état (compatibilité, états des connexions, des accessoires, collimateur, gaine d'éjection, télécommandes mécaniques...)

- met en place les moyens matériels et humains nécessaires à la bonne réalisation des contrôles demandés et en particulier procède au choix :

- du mode opératoire en proscrivant tout travail isolé

- du nombre et de la composition des équipes ; les équipes seront constituées d'un radiologue titulaire du CAMARI en cours de validité a minima

- du matériel adapté à l'opération (check list du matériel d'intervention et du matériel de protection) en s'assurant de son bon état (état des connexions, des accessoires, collimateur, gaine d'éjection, télécommandes mécaniques...) et de sa compatibilité...

- La PCR estime (ou valide la détermination de) la dosimétrie prévisionnelle et valide la délimitation de la zone d'opération prévisionnelle.

- Lors de la constitution des équipes, l'employeur tient compte de la dosimétrie des radiologues, de la validité de leur CAMARI et de l'autorisation de transport, des formations obligatoires suivies (formations à la sécurité et à la radioprotection)

- Elle communique à ses salariés toutes les informations nécessaires à la préparation de ses travaux

Il est rappelé que le travail isolé est interdit.

Si le $2^{\mathrm{e}}$ intervenant est titulaire également du CAMARI, cela donne une sécurité supplémentaire en cas de situation accidentelle dans laquelle un aide manipulateur se retrouvait en situation de devoir manipuler le projecteur.

\subsection{Le donneur d'ordre (DO)}

- Est garant de la sécurité et de la prévention des risques de son établissement envers tous les intervenants et responsable de la coordination générale des mesures de prévention et notamment de l'intervention des radiologues,

- S'assure de la configuration des lieux : accès, éclairage, ambiance de travail, points de rassemblement, local de stockage intermédiaire,...
Le Donneur d'Ordre est le seul à avoir autorité sur tous les intervenants présents sur le site susceptibles de créer une co-activité avec les radiologues (autres travaux, mais aussi conduite des installations, nettoyage, accompagnement des inspections... 


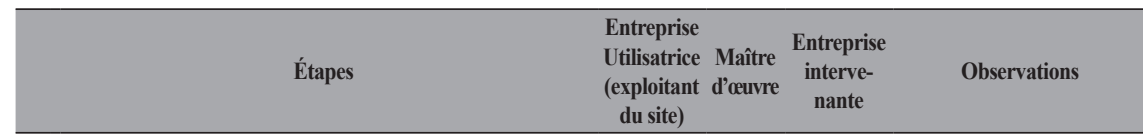

- S'assure que le personnel des postes de garde et de contrôle a été correctement formé et informé des risques liés à l'utilisation de rayonnements ionisants et connaît les procédures à appliquer afin de faciliter l'intervention des radiologues sur site.

- Il confirme la réalisation des travaux tels que prévus dans le permis de tir. avec un délai de prévenance raisonnable.

- Il s'assure de la configuration des lieux : éclairage, ambiance de travail, local de stockage intermédiaire, ...

- Il assure (ou fait assurer) la coordination des travaux

La PCR estime la dosimétrie prévisionnelle, vérifie le balisage prévisionnel et s'assure que le préréglage des alarmes des dosimètres opérationnels a bien été réalisé. Lors de la constitution des équipes, il devra être tenu compte :

- de la dosimétrie des radiologues

- de l'autorisation de transport (y compris classe 7)

- des formations obligatoires suivies.

En cas de travaux en hauteur, le DO veillera à ce que l'ensemble des besoins soit satisfait (éclairage, ...) et que l'entreprise d'échafaudage retenue soit capable de répondre aux besoins formulés notamment par les radiologues (manutention du gam, accessibilité et distance de repli).

\section{SITUATIONS SPÉCIFIQUES SUR SITE}

\section{INDUSTRIEL}

Selon l'intervention ou non d'une entreprise de maintenance, l'entreprise de radiographie industrielle interviendra :

- soit comme sous-traitant de l'entreprise de maintenance

- soit en sous-traitance directe de l'entreprise utilisatrice.

En fonction de la nature des travaux à réaliser, des délais différents minimums et des conditions particulières ont été définis.

\subsubsection{Maintenance préventive}

Délai entre la commande et le début des opérations de radiographie : 2 à 1 mois minimum
Dans l'esprit du décret 92-158, et surtout de la jurisprudence sur la délégation de pouvoir : il est le seul à avoir autorité et compétence et moyens.
R1

$$
\mathbf{R 2}
$$

R1

R1 R2
Le coordinateur doit avoir les connaissances nécessaires en Radioprotection. Dans le cas des EU sans PCR, l'EU peut solliciter une assistance externe.
EU MOE EI

$\mathbf{R} 1 \quad \mathbf{R} 2 \quad \mathbf{V} \quad \begin{aligned} & \text { L'EI le mentionne dans } \\ & \text { son offre. }\end{aligned}$




\begin{tabular}{lcccc}
\hline Étapes & $\begin{array}{c}\text { Entreprise } \\
\text { Utilisatrice Maître } \\
\text { (exploitant d'ouvre } \\
\text { du site) }\end{array}$ & $\begin{array}{c}\text { Entreprise } \\
\text { interve- } \\
\text { nante }\end{array}$ & Observations \\
\hline 3.2.2 Maintenance curative & EU & MOE & EI & \\
\hline
\end{tabular}

\section{Le délai minimum entre la commande et le début des opérations de radiographie varie selon l'urgence de la maintenance à réaliser :}

a) peu urgente : maintenance qui ne génère pas de dangers pour les hommes et le matériel : 1 mois

b) relativement urgente : maintenance qui ne nécessite pas une intervention rapide : 1 semaine à $48 \mathrm{~h}$

c) urgente (situation exceptionnelle présentant un danger immédiat pour les hommes et/ou les installations) : dans la journée avec mise en place de mesures compensatoires qui sont la mise en œuvre des moyens nécessaires pour assurer un même niveau de qualité de l'analyse de risque qu'en situation non dégradée (le niveau d'exigence doit rester identique en toutes circonstances).

\section{Pour la situation d'urgence : pour le donneur} d'ordre

Il choisit une entreprise de radiographie référencée ( $c f$. consultation).

Il crée une fiche d'urgence contenant :

- La mention « fiche d'urgence » précisant la situation exceptionnelle présentant un danger immédiat pour les hommes et/ou les installations

- Le travail à effectuer (programme des tirs, types de tirs, spécifications, atelier ou site)*

- Un plan du lieu d'intervention ou, en cas d'impossibilité majeure, un descriptif complet des lieux

- Le nom, le numéro de téléphone et la fonction du demandeur

- Le numéro de téléphone et la fonction de l'accueillant ayant autorité (c'est cette personne qui recevra les radiologues à leur arrivée sur le site)

- Les informations spécifiques essentielles à la sécurité (facteurs d'ambiance, conduite à tenir en cas d'accident, risques éventuellement liés à la co-activité, ....).

\section{Pour la situation d'urgence : pour l'entreprise de} radiographie industrielle

Elle communique à ses salariés toutes les informations nécessaires à la préparation de ses travaux

R1 R2

R C

R C

EU MOE EI

R1

R2 ou
C

* organe - Circuit concernés (soudure, ...) caractéristiques métallurgiques, dégradations recherchées, ... 


\begin{tabular}{lll}
\hline Étapes & $\begin{array}{l}\text { Entreprise } \\
\text { Utilisatrice Maître } \\
\text { (exploitant d'œuvre } \\
\text { du site) }\end{array}$ & $\begin{array}{c}\text { Entreprise } \\
\text { interve- } \\
\text { nante }\end{array}$ \\
\hline
\end{tabular}

- L'employeur choisit le mode opératoire, le nombre d'équipes, le matériel adapté (check list du matériel d'intervention et du matériel de protection) et s'assure de son bon état (compatibilité, états des connexions, des accessoires, collimateur, gaine d'éjection, télécommandes mécaniques...)

Elle complète la fiche d'urgence en renseignant :

- la partie dosimétrie prévisionnelle et balisage

- le nom de la PCR susceptible d'intervenir ainsi que son numéro de téléphone

- le nom des intervenants, la liste du matériel utilisé

\title{
Pour la situation d'urgence : à l'arrivée sur site
}

\begin{abstract}
À l'arrivée sur site, les radiologues sont reçus par l'accueillant désigné par le donneur d'ordre précédemment cité qui renseigne la partie «éléments de sécurité » (cheminements et sorties de secours, téléphones, boites à pharmacie, extincteurs, moyens d'urgence, douche/rince œil, ...).

Une visite préalable de chantier avec le DO est recommandée pour préciser les conditions d'interventions.

La fiche d'urgence doit être visée par le donneur d'ordre et le radiologue industriel.
\end{abstract}

V I $\quad$ R

EU MOE EI

R1 R2

R1 R2

R1 R2 R

\subsubsection{Grands arrêts ou arrêts d'unité}

EU MOE

EI

Durant cette période de plusieurs semaines pendant laquelle les unités de fabrication sont mises à disposition pour travaux de maintenance et/ ou de modernisation, les situations à risques vont principalement découler de la concomitance de plusieurs facteurs :

- risque de co-activité accru entre radiologues et autres corps de métier lié à l'aspect « travaux en continu »

- tirs croisés entre différentes équipes de radiologues (risque éventuellement accru si les radiologues n'appartiennent pas à la même entreprise)

- plage horaire de tirs très courte

- espaces confinés, ...

Chacun de ces facteurs de risque devra être réduit au niveau le plus bas (notamment par la mise en place de mesures organisationnelles visant à planifier le plus en amont possible les travaux de radiographie) afin de limiter au maximum le nombre de situations d'urgence. Pour ce faire, lors des grands arrêts, un « coordinateur de tirs radio » sera nommé par le donneur d'ordre qui lui octroiera les moyens en temps et en autorité.

$\mathbf{R}$

C

Pour mettre en œuvre cette coordination, l'EU peut solliciter une assistance externe. 


\begin{tabular}{|c|c|c|c|c|}
\hline Étapes & $\begin{array}{c}\text { Entreprise } \\
\text { Utilisatrice } \\
\text { (exploitant } \\
\text { du site) }\end{array}$ & Maître & $\begin{array}{c}\text { Entreprise } \\
\text { interve- } \\
\text { nante }\end{array}$ & Observations \\
\hline
\end{tabular}

La mission de coordination fera l'objet d'une mention spécifique dans le plan de prévention.

$\mathbf{R}$

Délai entre la commande et le début des opérations de radiographie : de $\mathbf{6}$ à 3 mois minimum afin de permettre aux entreprises de radiographie de s'organiser en amont.

\subsection{Coordination des tirs radios}

EU MOE

EI

Dans la mesure du possible :

- Prévoir une plage horaire spécifique aux tirs radio sans co-activité

$\mathbf{R}$

C

- Sélectionner une seule entreprise de radiographie industrielle pour l'ensemble des tirs à réaliser et limiter, en tout état de cause, le nombre d'entreprises intervenantes.

charges de l'intervention

- Évaluer, le plus précocement possible dans la journée, le volume global de tirs du soir et figer le plus tôt possible dans l'après midi le programme final des tirs et le communiquer à toutes les entreprises intervenantes et, en cas de co-activité résiduelle, aux entreprises concernées directement ou indirectement par l'intervention.

En collaboration avec les PCR si INB ou ICPE (DO et $\mathrm{EE})$, élaboration des plans de tirs et de balisage.

R $\quad$ C

\section{Mesures spécifiques en fonction du type de chantier}

\subsection{Les travaux en atelier}

Le travail en zone aménagée (bunker inclus) est fortement souhaitable et répondra aux exigences de l'arrêté zonage. Il apporte un maximum de garanties, évite le travail de nuit et répond à l'obligation de privilégier les protections collectives aux protections individuelles. La dérogation à cette organisation doit être justifiée et formalisée au travers du plan de prévention à partir du document interne dans lequel le chef d'établissement consigne la démarche qui lui a permis d'établir la délimitation des zones.

EU MOE EI

V

$\mathbf{R}$
Le niveau d'exposition des salariés aux rayonnements ionisants, lors de travaux en atelier reste significatif.

\subsection{Les travaux sur site industriel}

Délai entre la commande et le début des opérations de radiographie : 1 mois minimum
R1 


\begin{tabular}{lcccc}
\hline \multicolumn{1}{c|}{ Étapes } & $\begin{array}{c}\text { Entreprise } \\
\text { Utilisatrice Maître } \\
\text { (exploitant d'œurre } \\
\text { du site) }\end{array}$ & $\begin{array}{c}\text { Entreprise } \\
\text { interve- } \\
\text { nante }\end{array}$ & Observations \\
\hline $\begin{array}{l}\text { 4.3 Les travaux chez les chaudronniers } \\
\text { et les tuyauteurs }\end{array}$ & EU & MOE & EI & \\
\hline
\end{tabular}

Délai entre la commande et le début des opérations de radiographie : 1 semaine à 48 heures minimum

- Transmission du programme radio $48 \mathrm{H}$ minimum avant l'intervention. Le programme définit, outre le repérage technique : les nuances, épaisseurs, diamètres des équipements à radiographier

- Si un tir en bunker est possible, les tirs radios seront réalisés en horaires de travail de jour

- Si un tir en bunker n'est pas possible, l'intervention s'effectuera le plus tôt possible en fin d'après midi (après le départ des salariés de l'entreprise utilisatrice) sur une « zone unique et aménagée » à cet effet dans l'atelier du point de vue radioprotection. Un ensemble de dispositifs de radioprotection (écrans, ...) devront être installés sur cette zone aménagée afin de réduire au maximum les doses reçues par les radiologues, les salariés et le public.

- Regroupement, dans tous les cas où cela est possible, des équipements à contrôler dans une zone aménagée

- Prise en compte des spécificités de dernières minutes liées aux tirs devant être réalisés à l'extérieur (conditions climatiques, éclairage...)

- Identification des difficultés éventuelles et mise en place de mesures compensatoires (accessibilité, poids des pièces, difficultés particulières...)

- à chaque début de campagne de tirs (expliciter une campagne de tir)

- à chaque nouvelle équipe de radiologues

- à chaque changement significatif de l'environnement de travail (modification de l'atelier, déplacement de la zone unique et aménagée, pièces complexes à radiographier, ...)
$\mathbf{R}$

V

V L'EI doit vérifier

$\mathbf{R}$

\subsection{Cas des travaux sur chantier BTP}

Pour chaque chantier une étude préalable de faisabilité qui débouchera sur une proposition technique assortie

d'un plan de prévention stipulant :

- Les moyens d'accès spécifiques

- Le périmètre de sécurité

- La restriction de circulation avec prise d'arrêtés

- L'évacuation si nécessaire de tout ou une partie de la zone

- La surveillance renforcée lors des tirs 


\begin{tabular}{clc}
\hline Étapes & $\begin{array}{l}\text { Entreprise } \\
\text { Utilisatrice Maître } \\
\text { (exploitant d'ouvre } \\
\text { du site) }\end{array}$ & $\begin{array}{c}\text { Entreprise } \\
\text { interve- } \\
\text { nante }\end{array}$ \\
\hline
\end{tabular}

Une visite préalable de chantier est organisée

afin de procéder à l'examen des points suivants :

- Repérage des emplacements à contrôler

- Analyses des contraintes liées à l'environnement, touchant à la sécurité du public : délimitation des zones de sécurité, présence d'habitations ou de voies de circulation aux abords de la zone de tir

- problèmes d'accès

- problèmes liés au positionnement et à la fixation de la source et des films

- mise à disposition éventuelle de locaux pour le stockage et le développement sur chantier

\subsection{Cas des travaux sur chantier pipe-line sur sîtes} ruraux et urbains

Délai entre la commande et le début des opérations de radiographie : 3 mois à 1 mois

Une visite préalable de chantier est organisée afin de procéder à l'examen des points suivants :

- Repérage des emplacements à contrôler,

- Analyses des contraintes liées à l'environnement, touchant à la sécurité du public : délimitation des zones de sécurité, présence d'habitations ou de voies de circulation aux abords de la zone de tir,

- Utilisation des protections biologiques,

- Les problèmes d'accès (piste, dénivellation du terrain...),

- Formation pour l'utilisation des véhicules « $4 \times 4$ »,

- Fouilles à prévoir avec rampes d'accès pour pénétrer, et vérifier le talutage et le blindage,

- Les problèmes liés aux manutentions des crawleurs et sources,

- Vérifier la stabilité du tronçon à contrôler,

- Prise en compte des spécificités liées aux tirs devant être réalisés à l'extérieur (conditions climatiques...),

- La mise à disposition éventuelle de locaux pour le stockage du matériel et du laboratoire sur chantier,

On privilégiera les tirs en journée. La plage horaire doit tenir compte des risques à la fois pour les radiologues mais aussi de l'environnement des tirs (zones de passages, horaires d'affluence, ...)

Délai entre la commande et le début des opérations de radiographie : 3 mois à 1 mois
Les orientations des expositions les plus sécuritaires sont R C C déterminées selon la configuration des lieux par les intervenants CAMARI.
EU MOE EI
La délimitation des zones de travail est de la responsabilité de l'Entreprise Utilisatrice.

R1 R2 C
L'EU doit communiquer toutes les informations nécessaires (plans, ...). L'EU contrôle les accès, l'EI applique les consignes.

$\mathbf{R}$

C R

R

$\mathbf{R}$

$\mathbf{R}$

R

C

L'EU gère les

R1 R2 C co-activités et est responsable de la coordination 


\begin{tabular}{lccc}
\hline Étapes & $\begin{array}{c}\text { Entreprise } \\
\text { Utilisatrice Maître } \\
\text { (exploitant d'œuvre } \\
\text { du site) }\end{array}$ & $\begin{array}{c}\text { Entreprise } \\
\text { interve- } \\
\text { nante }\end{array}$ & Observations \\
\hline 4.6 Transport & EU & MOE & EI
\end{tabular}

Les gammagraphes sont pour la plupart des colis de type $\mathrm{B}(\mathrm{U})$, donc soumis à l'agrément de l'Autorité de sûreté nucléaire. Il existe également des gammagraphes de type $\mathrm{A}$ (colis de type $\mathrm{B}(\mathrm{U})$ déclassés en type A) qui n'ont pas de certificat d'agrément délivré par l'ASN pour le transport mais doivent avoir un certificat de conformité au colis de type A émis par le concepteur ou le propriétaire de l'emballage.

\section{PRESCRIPTIONS GÉNÉRALES}

Le transport n'est autorisé que si les dispositifs de verrouillage sont en position de fermeture, clé de sécurité retirée. Le transport avec clé de sécurité sur l'appareil est interdit.

Si le transport est effectué par le titulaire de l'autorisation ou un préposé titulaire du CAMARI, la clé peut être conservée par le chauffeur. Sinon, la clé doit faire l'objet d'une expédition distincte.

Si les transports sont effectués par une autre personne que le titulaire de l'autorisation ou un préposé (titulaire du CAMARI), l'entreprise doit avoir un système qualité certifié ISO 9001 version 2000 pour le transport.

Les conducteurs doivent suivre un cours de spécialisation formalisé par un certificat de formation du conducteur classe 7 (transport matières radioactives) avec recyclage tous les 5 ans. L'ensemble du personnel pouvant être amené à intervenir dans les opérations de transport doit suivre une formation de sensibilisation des dangers des rayonnements ionisants.

Le prévisionnel de dose doit tenir compte du transport. La société doit avoir un conseiller à la sécurité titulaire d'un certificat de qualification professionnelle. Une protection en plomb est positionnée (par exemple dans sa caisse de transport) entre le gammagraphe et le conducteur ainsi que le passager.

La caisse sera positionnée dans le véhicule de manière la plus éloignée des occupants.

Chaque intervention fait l'objet d'une fiche d'intervention.

À sa prise de poste les radiologues doivent

- porter deux dosimètres : passif et opérationnel conforme avec alarme sonore et étalonné.

- être classés aptitude médicale de catégorie A.

- détenir un radiamètre. 


\begin{tabular}{|c|c|c|c|c|}
\hline Étapes & $\begin{array}{c}\text { Entreprise } \\
\text { Utilisatrice } \\
\text { (exploitant } \\
\text { du site) }\end{array}$ & $\begin{array}{l}\text { Maître } \\
\text { t'œuvre }\end{array}$ & $\begin{array}{c}\text { Entreprise } \\
\text { interve- } \\
\text { nante }\end{array}$ & Observations \\
\hline
\end{tabular}

Pour chaque chantier, l'entreprise de radiographie industrielle doit clairement identifier le responsable de l'équipe des radiologues. Celui-ci doit s'assurer de l'applicabilité des documents de travail qui lui ont été délivrés et de leur mise en œuvre sur le chantier.

Les contrôles radiographiques sont réalisés dans les plages horaires définies par le donneur d'ordre.

R V

\subsection{Les documents de travail}

Le responsable de l'équipe des radiologues s'assure qu'ils disposent bien des documents nécessaires à l'intervention :

- Fiche d'intervention validée

- Plan de balisage validé

- Programme de tir

- Consignes, procédures et documents de travail nécessaires à l'intervention (sécurité et technique)

\subsection{Le balisage préalable}

Le balisage est réalisé au moyen de bandes précisant le danger d'exposition et complété d'un affichage indiquant l'interdiction de franchissement.

Les lampes à éclats, disposées au droit du balisage ou des points de passage,

offrent un meilleur repérage visuel du balisage et peuvent constituer des moyens d'avertissement efficaces, et sont à ce titre fortement recommandées. La mise en place du balisage et des moyens d'avertissement indiqués au plan de balisage (défini sur la base du plan de situation des installations, validé par la PCR de l'entreprise de radiographie industrielle et communiqué à l'entreprise utilisatrice) est effectuée sous la gouverne du responsable de l'équipe de radiographie.

La mise en place du balisage et des moyens d'avertissement indiqués au plan de balisage (défini sur la base du plan de situation des installations, si besoin validé par la PCR de l'EI et communiqué à l'EU) est effectuée sous la gouverne du responsable de l'équipe de radiographie.

En cas de difficulté de mise en œuvre du balisage ou de moyens d'avertissement prévus sur le plan (inexactitude, imprécision mineure entre le plan de situation et la réalité du terrain, mesures prévues insuffisantes), il appartient au responsable de l'équipe de radiographie de définir les corrections mineures à effectuer et d'informer le représentant de l'EU préalablement au début des tirs

EU MOE EI

$\begin{array}{lll}\text { V } & \text { V }\end{array}$

EU MOE EI

On peut utiliser des bandes répondant à la norme NF M 60-103

Les orientations des expositions les plus sécuritaires sont déterminées selon la configuration des lieux par les intervenants CAMARI 


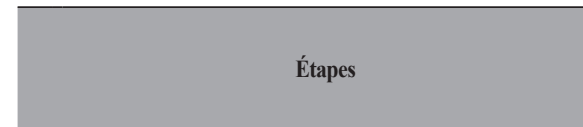

Les corrections apportées sont spécifiées sur le plan de balisage et celui-ci transmis à la PCR de l'EI et à celle de l'EU (si INB ou ICPE), et/ou le représentant désigné pour prise en compte dans le cadre du retour d'expérience et vérification de la non co-activité.

Ce processus vise une amélioration permanente des conditions de préparation des interventions mais ne peut porter que sur des modifications simples de mise en œuvre (rajout de la condamnation d'un accès oublié, déplacement d'un balisage par rapport à des limites géographiques plus aisées à réaliser et/ou surveiller). Dans tous les cas elles ne peuvent se faire au préjudice des dispositions réglementaires portant sur les débits de dose horaire ou des dispositions particulières spécifiées explicitement sur la fiche d'intervention et les documents associés (plan de prévention, analyse de risques, ...)

\begin{tabular}{|c|c|c|}
\hline $\begin{array}{l}\text { Entreprise } \\
\text { Utilisatrice Maître } \\
\text { (exploitant d'œuvre } \\
\text { du site) }\end{array}$ & $\begin{array}{l}\text { Entreprise } \\
\text { interve- } \\
\text { nante }\end{array}$ & Observations \\
\hline $\mathbf{R}$ & C & $\begin{array}{l}\text { Si des accès ou des } \\
\text { locaux supplémentaires } \\
\text { sont impactés, il est } \\
\text { recommandé } \\
\text { de refaire l'analyse } \\
\text { de risque, et donc } \\
\text { la fiche d'intervention. }\end{array}$ \\
\hline
\end{tabular}

Cette démarche est propre à l'installation. Les modifications devant profiter à toutes les entreprises, c'est à l'EU de capitaliser ce REX
Si des accès ou des sont impactés, il est la fiche d'intervention.

\subsection{Installation du matériel de radiographie industrielle}

Le montage du matériel de radiographie industrielle doit se faire dans un endroit sécurisé du chantier et préalablement rangé (débarrassé de tout objet inutile susceptible de présenter un risque pour les radiologues et/ou de générer des rayonnements secondaires). Les conditions d'éclairement de cet endroit doivent être suffisantes afin de garantir la bonne mise en place du matériel.

Avant assemblage du matériel, les radiologues doivent vérifier l'état et les connexions des accessoires (collimateur, gaine d'éjection, télécommande mécanique) avant de procéder à l'éjection.

Tout élément non conforme ou détérioré ne doit pas être utilisé, il doit être remis à l'employeur le jour même afin d'être réparé.

Avant toute éjection s'assurer qu'aucune personne ne stationne dans la zone. de façon à garantir l'entière intégrité du matériel, notamment :

- Le gam doit être obligatoirement situé dans un endroit stabilisé et protégé de son environnement.

- En cas de tirs radiographiques sur échafaudage ou en hauteur, le conteneur doit être arrimé de façon à éviter toute chute accidentelle.

- Les appareils (gam ou générateur X) ne doivent jamais être laissés sans surveillance. 


\begin{tabular}{|c|c|c|c|c|}
\hline Étapes & $\begin{array}{c}\text { Entreprise } \\
\text { Utilisatrice } \\
\text { (exploitant } \\
\text { du site) }\end{array}$ & $\begin{array}{l}\text { Maître } \\
\text { t'œuvre }\end{array}$ & $\begin{array}{c}\text { Entreprise } \\
\text { interve- } \\
\text { nante }\end{array}$ & Observations \\
\hline
\end{tabular}

- L'appareil ne doit être déplacé, y compris à l'intérieur des limites du balisage, que s'il est verrouillé, clé de sécurité dégagée et séparée de l'appareil.

\subsection{Vérification du balisage}

Lors de la première éjection, l'un des membres de l'équipe s'assure à l'aide d'un radiamètre que le débit de dose aux limites de balisage est inférieur ou égal aux limites définies (plan de prévention, analyse de risques, ...).

Des modifications successives du balisage peuvent être prévues par le plan de balisage pour s'adapter aux différentes situations de tir dans le cadre d'une même intervention. Cette vérification sera effectuée à chaque modification.

En cas de dépassement de débit de dose aux limites de balisage qui conduirait à une modification importante de celui-ci pour obtenir la conformité aux limites fixées, le responsable de l'équipe de radiographie industrielle en informe le représentant de l'EU et sa PCR.

L'intervention ne pourra être poursuivie qu'après nouvelle analyse et définition d'un nouveau plan de balisage réalisé et validé par la PCR de l'EI et communiqué à celle de l'EU. Cette nouvelle analyse sera obligatoirement sur le lieu des tirs ou dans les locaux de la société de radiographie.
V

$\mathbf{R}$

V

$\mathbf{R}$

V

$\mathbf{R}$

V

$\mathbf{R}$

\subsection{Points de replis}

E

MOE

EI

Lors de la réalisation des tirs radiographiques, les radiologues s'assurent au moyen de leur radiamètre du très faible débit de dose aux points de replis définis par la PCR de l'EI.

\subsection{Situations incidentelles/accidentelles}

EU MOE

EI

Toute situation incidentelle/accidentelle est déclarée immédiatement à la PCR de l'EI et au représentant

de l'EU et/ou à sa PCR notamment :

- Chute du gam

- Source bloquée à l'extérieur du gam

- Source bloquée à l'intérieur du gam

- Exposition externe accidentelle

- Source perdue

- Écrasement de la gaine d'éjection 


\begin{tabular}{lll}
\hline Étapes & $\begin{array}{l}\text { Entreprise } \\
\text { Utilisatrice Maître } \\
\text { (exploitant d'œuvre } \\
\text { du site) }\end{array}$ & $\begin{array}{c}\text { Entreprise } \\
\text { interve- } \\
\text { nante }\end{array}$ \\
\hline
\end{tabular}

- Plus généralement, tout événement extérieur pouvant entraîner la dégradation du projecteur, de la gaine, du porte source

- Accident de transport

Ces situations et la conduite à tenir sont définies dans des consignes ou procédures écrites de l'EI, documents qui font partie des documents de travail dont disposent les radiologues sur le chantier et leur sont présentées dans le cadre de la formation à la sécurité. Elles font l'objet d'un retour d'expérience ( $c f$. paragraphe suivant).

\title{
5.7 Repli de chantier
}

C ou V

$\mathbf{R}$

\begin{abstract}
À la fin de chaque poste de travail, les radiologues doivent verrouiller l'appareil en retirant la clef et s'assurent que le porte source se trouve bien dans le projecteur avec le radiamètre.

L'appareil peut être entreposé dans un local intermédiaire mis à disposition par le DO si l'intervention dure plusieurs jours.

L'appareil sera arrimé dans le véhicule.

Les radiologues après avoir retiré le balisage afin de remettre le chantier en libre accès formalisent la fin de l'intervention auprès du représentant de l'EU.
\end{abstract}

\section{EU MOE EI}

\subsection{Fin de poste de travail}

- Relevé des mesures de dosimétries opérationnelles.

- Retrait des deux dosimètres et rangement dans l'emplacement prévu à cet effet.

\subsection{Retour d'expérience}

Un compte rendu d'intervention écrit est rédigé par le chef d'équipe après chaque opération et peut être annexé au document unique d'évaluation des risques.

Ce compte rendu devra contenir au minimum les renseignements suivants :

- Date d'intervention

- Heure d'intervention

- Lieu d'intervention

- Lieu d'intervention

- Nom des radiologues

- Nom du donneur d'ordre

- Le prévisionnel dosimétrique
EU MOE EI

EU MOE EI

Le compte-rendu d'intervention peut figurer dans le cahier des charges et dans la commande.

Le résultat du suivi dosimétrique fait partie de l'analyse demandée R par les articles R231-94 du décret 2003-296. Il convient de respecter la confidentialité. 


\begin{tabular}{|c|c|c|c|c|}
\hline Étapes & $\begin{array}{c}\text { Entreprise } \\
\text { Utilisatrice } \\
\text { (exploitant } \\
\text { du site) }\end{array}$ & Maître & $\begin{array}{c}\text { Entreprise } \\
\text { interve- } \\
\text { nante }\end{array}$ & Observations \\
\hline
\end{tabular}

- Les résultats de la dosimétrie opérationnelle

- Relations avec donneurs d'ordre

- Heures d'attente

- Matériels défaillants

- Difficultés rencontrées

- Incidents / accidents

Le retour d'expérience s'attachera notamment :

- aux travaux qui n'auront pas pu être réalisés compte tenu :

- des écarts importants constatés au regard des conditions de travail initialement prévues (échafaudage non adapté, éclairage insuffisant, plan de zone erroné, accès difficile, ambiance de travail non spécifiée, éléments de sécurité manquants...)

- des organisations du travail modifiées (modification du programme de tirs, temps prévu insuffisant, problèmes

de communication, problème de co-activité...).

- aux opérations qui se sont réalisées mais pour lesquelles il aura été relevé des écarts entre les doses reçues et les doses prévisionnelles.

Ces écarts donneront lieu à une analyse approfondie des causes du dysfonctionnement pour permettre la mise en œuvre de mesures correctives ou préventives et la mise à jour du document unique. L'analyse doit être menée conjointement par le donneur d'ordre et l'entreprise de radiographie industrielle.
Le résultat du suivi dosimétrique fait partie de l'analyse demandée par les articles R231-94 du décret 2003-296. Il convient de respecter la confidentialité.
L'analyse peut être faite pour les petits chantiers, les chantiers urgents, ...

\section{Formation et information du personnel}

Toute personne susceptible d'être exposée aux rayonnements ionisants (zone surveillée, zone contrôlée ou zone d'opération) doit bénéficier d'une formation obligatoire à la radioprotection organisée par le chef d'entreprise.

Cette formation, essentielle pour les radiologues (et distincte de celle délivrée dans le cadre de la préparation à l'examen CAMARI), à laquelle il est important d'associer le médecin du travail et la PCR, doit : 


\begin{tabular}{|c|c|c|c|c|}
\hline Étapes & $\begin{array}{c}\text { Entreprise } \\
\text { Utilisatrice } \\
\text { (exploitant } \\
\text { du site) }\end{array}$ & $\begin{array}{l}\text { e Maître } \\
\text { t d'œuvre }\end{array}$ & $\begin{array}{c}\text { Entreprise } \\
\text { interve- } \\
\text { nante }\end{array}$ & Observations \\
\hline
\end{tabular}

- Être renouvelée périodiquement, au moins tous les trois ans et à chaque fois que nécessaire (modification des conditions de travail, évolution des consignes de sécurité sur la base du retour d'expérience, évolution de la réglementation...)

- Porter sur les risques liés à l'exposition aux rayonnements ionisants, les procédures générales de radioprotection ainsi que les règles de prévention et de protection propres à l'entreprise.

- Être très pratique et adaptée aux situations susceptibles d'être rencontrées par le salarié.

- Permettre au salarié notamment d'adapter son comportement, sans risques pour lui et pour autrui, aux situations particulières rencontrées, de connaître la conduite à tenir en cas d'incident ou d'accident.

- Être complétée, si nécessaire, lors des accueils sécurité sur sites ou lors de la transmission des données du plan de prévention ou des PPSPS, par des procédures particulières de radioprotection mises en œuvre dans les différents établissements ou chantiers

- Être suivie, pour les nouveaux embauchés, par une phase de " compagnonnage » ou de tutorat dans l'entreprise.

- Être complétée, pour les femmes, d'une information sur les préconisations spécifiques concernant les risques liés aux rayonnements pour la grossesse.

Toute personne utilisatrice d'appareils de gammagraphie ou de générateurs à rayons $\mathrm{X}$ doit être titulaire d'un CAMARI en cours de validité.

\section{Suivi dosimétrique et médical}

Les radiologues industriels sont classés en catégorie A par l'employeur après avis

du médecin du travail.

Compte tenu de la difficulté du suivi médical et dosimétrique des salariés intérimaires ou en CDD, il ne sera recouru à ce type de contrat que de manière limitée. Dans ces cas-là, l'employeur devra s'assurer du respect des dispositions réglementaires relatives au suivi médical et dosimétrique des salariés concernés. 


\begin{tabular}{lcccc}
\hline Étapes & $\begin{array}{c}\text { Entreprise } \\
\text { Utilisatrice Maître } \\
\text { (exploitant d'ouvre } \\
\text { du site) }\end{array}$ & $\begin{array}{c}\text { Entreprise } \\
\text { interve- } \\
\text { nante }\end{array}$ & Observations \\
\hline 7.1 Suivi dosimétrique & EU & MOE & EI & \\
\hline
\end{tabular}

Les radiologues doivent être équipés d'un dosimètre passif nominatif (avec numéro d'identification) et d'un dosimètre opérationnel conformes à la réglementation; le dosimètre opérationnel sera muni d'une alarme.

La dosimétrie opérationnelle est un outil de prévention du risque radiologique qui permet, opération par opération, de vérifier qu'il n'y ait pas de dépassement de la dosimétrie prévisionnelle. La dosimétrie passive, quant à elle, donne des résultats différés et participe donc à l'évaluation du risque. Elle permet ainsi un suivi médical adapté.

La visite médicale est également l'occasion pour le radiologue d'une discussion avec le médecin du travail sur les résultats de sa dosimétrie.

\section{Dosimétrie passive}

L'organisme chargé de la dosimétrie passive transmet les résultats :

- mensuellement au médecin du travail

- annuellement au salarié

- à la PCR sur sa demande pour procéder à

l'évaluation prévisionnelle des doses (sur les 12 derniers mois maxi)

L'IRSN centralise ces résultats dans la base de

données SISERI et en organise l'accès aux médecins du travail, aux PCR et aux travailleurs ainsi qu'aux médecins désignés par les salariés.

\section{Dosimétrie opérationnelle (active)}

La PCR met en œuvre la dosimétrie opérationnelle et transmet les résultats :

- mensuellement au médecin du travail

- mensuellement au chef d'entreprise

- hebdomadairement à l'IRSN en vue de les centraliser dans la base de données SISERI

- mensuellement à chaque salarié

En cas de discordance entre les résultats de la dosimétrie passive et de la dosimétrie opérationnelle, le médecin du travail détermine la dose reçue par le travailleur.

Après enquête, et consolidation des résultats, il retiendra la valeur la plus élevée, même s'il s'agit de la dosimétrie opérationnelle. 


\begin{tabular}{lll}
\hline Étapes & $\begin{array}{l}\text { Entreprise } \\
\text { Utilisatrice Maître } \\
\text { (exploitant d'œuve } \\
\text { du site) }\end{array}$ & $\begin{array}{c}\text { Entreprise } \\
\text { interve- } \\
\text { nante }\end{array}$ \\
\hline
\end{tabular}

7.2 Situations d'alerte dosimétrique

Dès lors que la dosimétrie passive ou opérationnelle d'un radiologue atteint plusieurs mSv sur un mois ou sur une opération ponctuelle, cette situation doit entraîner :

- une enquête de l'employeur et menée par la PCR en collaboration avec le radiologue et le médecin du travail. Elle cherchera à déterminer l'origine de l'exposition et à mettre en place des mesures préventives; les résultats de cette enquête et des mesures prises feront l'objet d'un REX

- une consultation du radiologue concerné auprès de son médecin du travail.

$\underline{\text { Risque de dépassement des limites réglementaires }}$ Quand il y a risque de dépassement des limites réglementaires et demande de lecture du dosimètre passif en urgence, le radiologue ne doit pas être exposé aux rayonnements ionisants le temps d'avoir confirmation des doses effectivement reçues.

\section{Dépassement des valeurs limites réglementaires}

En cas de dépassement des valeurs limites

réglementaires, seront informés :

- l'employeur

- le médecin du travail

- le salarié

- la PCR

- le CHSCT (ou à défaut DP)

- les inspections concernées (inspection du travail et inspection en radioprotection)

- la CRAM

\section{EU MOE EI}

L'entreprise peut se fixer un seuil mensuel de $\mathrm{x}$ $\mathrm{mSv}$.

\subsection{Suivi médical}

Les radiologues bénéficient d'une surveillance médicale renforcée et doivent à ce titre passer une visite médicale au moins une fois par an. La fiche individuelle d'exposition, mise à jour par l'employeur avant chaque visite médicale, sera remise par le radiologue au médecin du travail à cette occasion. 


\begin{tabular}{|c|c|c|c|}
\hline Étapes & $\begin{array}{l}\text { Entreprise } \\
\text { Utilisatrice Maître } \\
\text { (exploitant d'œuvre } \\
\text { du site) }\end{array}$ & $\begin{array}{c}\text { Entreprise } \\
\text { interve- } \\
\text { nante }\end{array}$ & Observations \\
\hline $\begin{array}{l}\text { Il est recommandé que le médecin du travail suive } \\
\text { une formation relative au risque radiologique. } \\
\text { Outre les éléments devant figurer réglementairement } \\
\text { dans le dossier médical des radiologues } \\
\text { (données dosimétriques, résultats des examens } \\
\text { complémentaires, double de la fiche individuelle } \\
\text { d'exposition), seront également conservés tous } \\
\text { les documents relatifs aux alertes dosimétriques } \\
\text { précédemment définies. } \\
\text { Chaque radiologue doit être en possession de sa carte } \\
\text { individuelle de suivi médical (carte professionnelle) } \\
\text { délivrée par le médecin du travail. } \\
\text { À son départ de l'entreprise, quel qu'en soit le } \\
\text { motif, le radiologue doit recevoir une attestation } \\
\text { d'exposition, remplie par l'employeur et le médecin } \\
\text { du travail, afin de pouvoir bénéficier } \\
\text { d'une surveillance médicale post-professionnelle. }\end{array}$ & & $\mathbf{R}$ & \\
\hline
\end{tabular}

